ORNL/TM-2014/215

Energy and Transportation Science Division $\&$

Facilities Management Division

\title{
DERIVATION OF BUILDING ENERGY USE INTENSITY \\ TARGETS FOR ASHRAE STANDARD 100 \\ AUGUST 31, 2011
}

Terry R. Sharp

Date Published: June 2014

Prepared by

OAK RIDGE NATIONAL LABORATORY

Oak Ridge, Tennessee 37831-6283

managed by

UT-BATTELLE, LLC

for the

U.S. DEPARTMENT OF ENERGY

under contract DE-AC05-00OR22725

Approved for public release;

distribution is unlimited. 


\section{DOCUMENT AVAILABILITY}

Reports produced after January 1, 1996, are generally available free via the U.S.

Department of Energy (DOE) Information Bridge.

Web site http://www.osti.gov/bridge

Reports produced before January 1, 1996, may be purchased by members of the public from the following source.

National Technical Information Service

5285 Port Royal Road

Springfield, VA 22161

Telephone 703-605-6000 (1-800-553-6847)

TDD 703-487-4639

Fax 703-605-6900

E-mail info@ntis.gov

Web site http://www.ntis.gov/support/ordernowabout.htm

Reports are available to DOE employees, DOE contractors, Energy Technology Data Exchange (ETDE) representatives, and International Nuclear Information System (INIS) representatives from the following source.

Office of Scientific and Technical Information

P.O. Box 62

Oak Ridge, TN 37831

Telephone 865-576-8401

Fax 865-576-5728

E-mail reports@osti.gov

Web site http://www.osti.gov/contact.html

This report was prepared as an account of work sponsored by an agency of the United States Government. Neither the United States Government nor any agency thereof, nor any of their employees, makes any warranty, express or implied, or assumes any legal liability or responsibility for the accuracy, completeness, or usefulness of any information, apparatus, product, or process disclosed, or represents that its use would not infringe privately owned rights. Reference herein to any specific commercial product, process, or service by trade name, trademark, manufacturer, or otherwise, does not necessarily constitute or imply its endorsement, recommendation, or favoring by the United States Government or any agency thereof. The views and opinions of authors expressed herein do not necessarily state or reflect those of the United States Government or any agency thereof. 


\title{
Derivation of Building Energy Use Intensity Targets for ASHRAE Standard 100
}

\author{
Terry R. Sharp \\ Oak Ridge National Laboratory \\ August 31, 2011
}

The steps to develop the building energy use intensity targets for ASHRAE Standard 100 are outlined below. These analyses were conducted by Oak Ridge National Laboratory (ORNL) in collaboration with the ASHRAE Standard 100 committee and Dr. Alexander Zhivov, the subcommittee chair responsible for targets development.

Step 1: Generate Site-Energy-Based Building Total Energy Use Intensities by Building Type and Climate Zone

In this step, national building total energy use intensities (EUIs; units are kBtu/sqft-yr) are derived via analysis of the CBECS of 2003 and the RECS of 2005. The EUIs are site-energy based ${ }^{1}$ and are national median values. The building EUI values are derived based on building types as classified by the "PBAPLUS8" variable in the CBECS database and the "TYPEHUQ" variable in the RECS database. This classification yielded over 50 different building types for the analysis (48 commercial and 5 residential). A goal of this step was to develop building EUIs by climate zone because EUIs for any given building type differ significantly depending on their climatological location.

The CBECS database, via a simple parsing method, was found inadequate to provide reliable EUI values by climate zone for a large number of commercial U.S. building types. This was primarily due to insufficient sample size when the data were parsed by building type and climate zone. A similar problem was noted by Griffin, et al. As a result, an alternative method was utilized to derive EUIs by climate zone. Zonal EUI ratios (EUI for climate zone/EUI national) were provided from building simulation modeling performed by the National Renewable Energy Laboratory (NREL; Deru, et al.) for 16 different climate zones (climate zones are shown in Figure 1). ${ }^{2,3}$ These ratios are shown below and were used to derive zonal EUIs by building type by multiplying them by the CBECS national median EUIs. This step produced the site-based building total EUIs by building type and climate zone shown in Table 1. A similar method was used to

\footnotetext{
1 "Site" energy use refers to energy use measured at the building, usually by the electric, natural gas, and other energy meters.

2 In the zonal EUI ratio calculation, consistent with the basis for the "EUI for climate zone" term, the "EUI national" term was also based on NREL simulation results. It is not identical to a CBECS or RECS national value.

${ }^{3}$ The 16 different climate zones referenced in this document consists of the 8 zones shown in Figure 1 (color coded) split into the moist (A), dry (B), and marine (C) regions as delineated in the figure. Note that the small climate zone (both in geographical area and number of buildings) identifiable as Climate Zone 7B in Figure 1 was not analyzed by NREL and is not represented in this analysis. These are further described by Briggs, et al.
} 


\begin{tabular}{|c|c|c|c|c|c|c|c|c|c|c|c|c|c|c|c|c|}
\hline \multicolumn{17}{|c|}{ Ratios of climate zonal EUI to average of all zonal EUls by building type } \\
\hline $\begin{array}{l}\text { ASHRAE Climate } \\
\text { Zone: }\end{array}$ & $1 \mathrm{~A}$ & $2 A$ & 2B & 3A & \begin{tabular}{|c|} 
3B- \\
Coast
\end{tabular} & $\begin{array}{c}\text { 3B- } \\
\text { Other }\end{array}$ & 3C & 4A & 4B & 4C & 5A & 5B & 6A & 6B & 7 & 8 \\
\hline Building Type & \multicolumn{16}{|c|}{ Climate Zone EUI Ratio } \\
\hline All Office & 0.91 & 0.94 & 0.91 & 0.97 & 0.76 & 0.90 & 0.78 & 1.06 & 0.92 & 0.94 & 1.12 & 0.97 & 1.25 & 1.10 & 1.35 & 1.89 \\
\hline Large Office & 0.88 & 0.95 & 0.88 & 0.98 & 0.80 & 0.89 & 0.81 & 1.07 & 0.88 & 0.98 & 1.07 & 0.91 & 1.15 & 1.01 & 1.21 & 1.63 \\
\hline Medium Office & 0.98 & 0.97 & 0.98 & 0.97 & 0.78 & & 0.77 & 1.05 & 0.91 & 0.93 & 1.11 & 0.96 & 1.24 & 1.09 & 1.33 & 1.86 \\
\hline Small Office & 0.87 & 0.90 & 0.89 & 0.95 & 0.71 & 39 & 0.75 & 1.06 & 0.96 & 0.91 & 1.19 & 1.06 & 1.36 & 1.21 & 1.50 & 2.17 \\
\hline Warehouse & 0.52 & 0.66 & 0.69 & 0.84 & 0.45 & 0.75 & 0.61 & 1.13 & 0.95 & 0.92 & 1.50 & 1.26 & 2.07 & 1.70 & 2.53 & 4.75 \\
\hline Stand-alone Retail & 0.85 & 0.87 & 0.85 & 0.91 & 0.65 & 0.83 & 0.73 & 1.03 & 0.90 & 0.90 & 1.17 & 1.01 & 1.35 & 1.20 & 1.50 & 2.16 \\
\hline Strip Mall & 0.89 & 0.89 & 0.87 & 0.94 & 0.69 & 86 & 0.77 & 1.08 & 0.94 & 0.96 & 1.24 & 1.06 & 1.43 & 1.27 & 1.60 & 2.29 \\
\hline Primary School & 0.98 & 0.97 & 0.93 & 0.97 & 0.79 & & 0.83 & 1.06 & 0.93 & 0.94 & 1.10 & 0.96 & 1.20 & 1.06 & 1.27 & 1.87 \\
\hline Secondary School & 0.91 & 0.90 & 0.89 & 0.92 & 0.66 & 0.86 & 0.74 & 1.06 & 0.88 & 0.95 & 1.15 & 0.96 & 1.33 & 1.14 & 1.46 & 2.16 \\
\hline Supermarket & 0.83 & 0.90 & 0.84 & 0.94 & 0.78 & 0.86 & 0.87 & 1.02 & 0.92 & 0.97 & 1.10 & 1.00 & 1.19 & 1.10 & \begin{tabular}{|l|}
1.29 \\
\end{tabular} & 1.62 \\
\hline Fast Food & 0.89 & 0.91 & 0.90 & 0.95 & 0.81 & 0. & 0.86 & 1.04 & 0.96 & 0.97 & 1.13 & 1.03 & 1.24 & 1.14 & \begin{tabular}{|l|}
1.34 \\
\end{tabular} & 1.70 \\
\hline Restaurant & 0.88 & 0.91 & 0.88 & 0.94 & 0.79 & 0.89 & 0.86 & 1.04 & 0.95 & 0.97 & 1.12 & 1.02 & 1.22 & 1.13 & \begin{tabular}{|l|l|}
1.33 \\
\end{tabular} & 1.68 \\
\hline Hospital & 1.02 & 1.03 & 1.00 & 1.01 & 0.96 & 0.99 & 0.94 & 1.03 & 0.92 & 0.97 & 1.00 & 0.91 & 1.02 & 0.94 & 1.03 & 1.19 \\
\hline Outpatient health care & 1.08 & 1.04 & 1.05 & 1.03 & 0.97 & & 0.86 & 1.02 & 0.98 & 0.91 & 0.98 & 0.96 & 1.00 & 0.97 & 0.99 & 1.12 \\
\hline Motel & 1.07 & 1.01 & 1.01 & 0.98 & 0.92 & 0.97 & 0.90 & 1.00 & 0.96 & 0.92 & 1.02 & 0.97 & 1.08 & 1.01 & 1.10 & 1.32 \\
\hline Hotel & 0.93 & 0.96 & 0.91 & 0.97 & 0.88 & 0.92 & 0.91 & 1.03 & 0.98 & 0.99 & 1.08 & 1.03 & 1.15 & 1.11 & 1.22 & 1.41 \\
\hline Mid-Rise Apartment & 0.78 & 0.82 & 0.82 & 0.92 & 0.61 & 0.84 & 0.78 & 1.12 & 0.93 & 1.04 & 1.27 & 1.07 & 1.45 & 1.28 & 1.65 & 2.30 \\
\hline
\end{tabular}

derive the zonal EUIs for residential building types using the RECS database (also listed in Table 1).

Step 2: Identify Representative $25^{\text {th }}$ Percentile Values by Climate Zone

The ASHRAE Standard 100 committee wanted to use the top (best) performing $25^{\text {th }}$ percentile of an EUI distribution for each building type as the energy target for buildings. In this respect (there are considerable differences in others), this approach is similar to the criteria that EPA uses for its highly recognized Energy Star designation awarded to commercial buildings. The challenge was to identify representative $25^{\text {th }}$ percentile values when there was no climate-zone EUI distribution available.

This was done by comparing the $25^{\text {th }}$ percentile values from the CBECS/RECS national distributions to the distribution medians - the $50^{\text {th }}$ percentile value (by building type of course). A simple ratio of the $25^{\text {th }}$ to $50^{\text {th }}$ percentiles was developed for each building type. These ratios were then multiplied by the climate-zone based EUIs developed in Step 1 to approximate a $25^{\text {th }}$ percentile EUI value in each climate zone.

At this point, we had established high-performance energy targets by building type for each climate zone. These results are summarized in Table 2.

Step 3: Develop Schedule Multipliers

Beyond floor area, another major driver of energy use in buildings is operating hours. While perhaps a minimal concern for residential buildings (we assume 24/7 operation for residential), they are a significant energy use driver for many types of commercial buildings. As a result, ORNL conducted an analysis to investigate the impact of schedule 
by building type and used that to develop a set of schedule multipliers that could be used to adjust building energy use intensities for schedule such that the comparison of a users building to Standard 100 targets could be a more reliable (i.e., a more "apple-to-apples") comparison. An example of this need is illustrated by comparing an EUI from an office building operated for three shifts to one that is operated for only one shift. All other influences including building size being the same, the three shift building would be expected to be considerably more energy intensive that the one shift building.

Derivation of schedule multipliers began with a graphic, histogram-based inspection of the weekly operating hours of all CBECS observations by building type (the national sample). From these, three dominant weekly operating hours categories emerged. They were 1) 50 or less weekly operating hours, 2) 168 operating hours, and 3) between 50 and 168 operating hours. Based on these, median national EUIs for the CBECS observations in each of these three operational categories were calculated by building type. These were then divided by the CBECS national medians by building type (for all operational categories) to derive ratios for shift multipliers by building type. The resulting shift multipliers are summarized in Table 3.

\section{REFERENCES}

1. Briggs, Robert S., et al., "Climate Classification for Building Energy Codes and Standards" by the Pacific Northwest National Laboratory March 2002.

2. Deru, Michael, et al., "U.S. Department of Energy Commercial Reference Building Models of the National Building Stock" by the National Renewable Energy Laboratory (NREL/TP-550046861, February 2011) available at http://www.nrel.gov/docs/fy11osti/46861.pdf.

3. Griffin, B., et al., "Methodology for Modeling Building Energy Performance Across the Commercial Sector" by the National Renewable Energy Laboratory (NREL/TP-550-41956, March 2008) available at http://www.nrel.gov/docs/fy08osti/41956.pdf.

4. U.S. Department of Energy, Energy Information Administration, Commercial Building Energy Consumption Survey (2003) and Residential Energy Consumption Survey (2005), http://www.eia.doe.gov/emeu/cbecs/ and http://www.eia.doe.gov/emeu/recs/. 


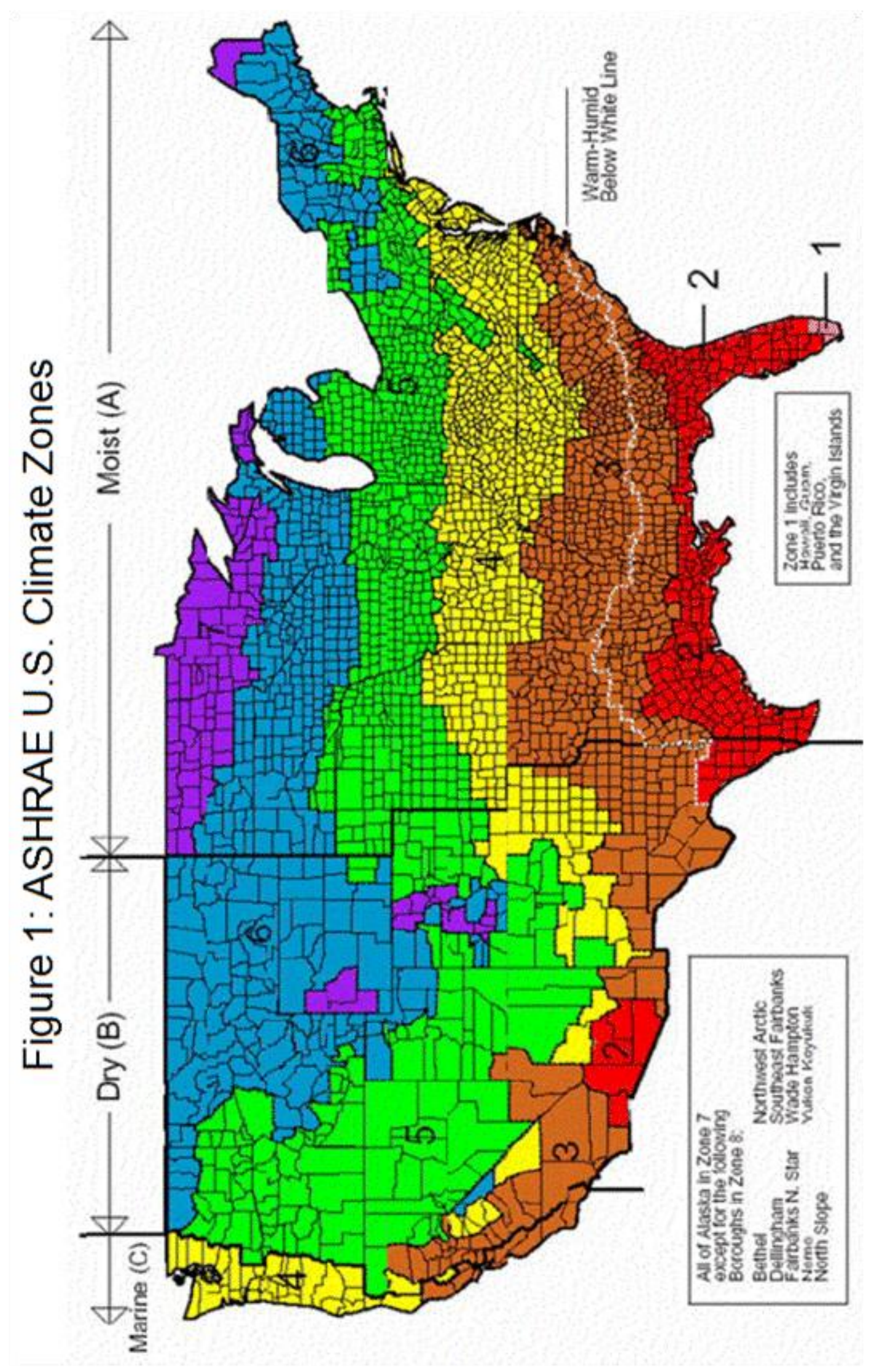


Figure 1: ASHRAE U.S. Climate Zones (cont.)

\begin{tabular}{|c|c|c|}
\hline Zone & Thermal Criteria & Representative U.S. City \\
\hline 1A & $5000<\mathrm{CDD} 10^{\circ} \mathrm{C}$ & Miami \\
\hline 2A & $3500<\mathrm{CDD} 10^{\circ} \mathrm{C} \leq 5000$ & Houston \\
\hline 2B & $3500<\mathrm{CDD} 10^{\circ} \mathrm{C} \leq 5000$ & Phoenix \\
\hline 3A & $2500<\mathrm{CDD} 10^{\circ} \mathrm{C} \leq 3500$ & Atlanta \\
\hline 3B-Coast & $2500<\mathrm{CDD} 10^{\circ} \mathrm{C} \leq 3500$ & Los Angeles \\
\hline 3B-Other & $2500<\mathrm{CDD} 10^{\circ} \mathrm{C} \leq 3500$ & Las Vegas \\
\hline 3C & $\mathrm{HDD} 18^{\circ} \mathrm{C} \leq 2000$ & San Francisco \\
\hline 4A & $\mathrm{CDD}^{\circ} \mathrm{C} \leq 2500 \mathrm{AND} \mathrm{HDD} 18^{\circ} \mathrm{C} \leq 3000$ & Baltimore \\
\hline 4B & $\mathrm{CDD} 10^{\circ} \mathrm{C} \leq 2500 \mathrm{AND} \mathrm{HDD} 18^{\circ} \mathrm{C} \leq 3000$ & Albuquerque \\
\hline 4C & $2000<\mathrm{HDD} 18^{\circ} \mathrm{C} \leq 3000$ & Seattle \\
\hline 5A & $3000<\mathrm{HDD} 18^{\circ} \mathrm{C} \leq 4000$ & Chicago \\
\hline 5B & $3000<\mathrm{HDD} 18^{\circ} \mathrm{C} \leq 4000$ & Denver \\
\hline 6A & $4000<\mathrm{HDD} 18^{\circ} \mathrm{C} \leq 5000$ & Minneapolis \\
\hline $6 \mathrm{~B}$ & $4000<\mathrm{HDD} 18^{\circ} \mathrm{C} \leq 5000$ & Helena \\
\hline 7.0 & $5000<\mathrm{HDD} 18^{\circ} \mathrm{C} \leq 7000$ & Duluth \\
\hline 8.0 & $7000<\mathrm{HDD} 18^{\circ} \mathrm{C}$ & Fairbanks \\
\hline
\end{tabular}

Notes:

1. Climate zones are more fully described in the references (Briggs, et al.; Deru, et al.)

2. Representative cities were used for simulation modeling. 


\begin{tabular}{|c|c|c|c|c|c|c|c|c|c|c|c|c|c|c|c|c|c|}
\hline \multicolumn{18}{|c|}{ Table 1: CBECS/RECS Total Site-Based Energy Use Intensities ${ }^{1}$} \\
\hline \multirow[b]{2}{*}{ Building Category } & ASHRAE Climate Zone: & $1 \mathrm{~A}$ & $2 A$ & 2B & 3A & \begin{tabular}{|c} 
3B- \\
Coast
\end{tabular} & $\begin{array}{c}\text { 3B- } \\
\text { Other }\end{array}$ & $3 C$ & 4A & 4B & 4C & $5 \mathbf{A}$ & $5 B$ & $6 \mathrm{~A}$ & 6B & 7 & 8 \\
\hline & Building Type & \multicolumn{16}{|c|}{ Total Site-Based Energy Use Intensity (site kBtu/yr-sqft) } \\
\hline Education & College/university & 88 & 88 & 86 & 89 & 64 & 83 & 72 & 103 & 86 & 92 & 112 & 93 & 129 & 111 & 142 & 210 \\
\hline Education & Elementary/middle school & 54 & 53 & 51 & 53 & 43 & 50 & 46 & 58 & 51 & 52 & 60 & 53 & 66 & 58 & 70 & 103 \\
\hline Education & High school & 64 & 64 & 63 & 65 & 47 & 61 & 53 & 75 & 63 & 67 & 81 & 68 & 94 & 81 & 103 & 153 \\
\hline Education & Other classroom education & 36 & 36 & 35 & 36 & 26 & 34 & 29 & 42 & 35 & 38 & 46 & 38 & 53 & 45 & 58 & 86 \\
\hline Education & Preschool/daycare & 70 & 68 & 66 & 69 & 56 & 64 & 59 & 75 & 66 & 66 & 78 & 68 & 85 & 75 & 90 & 133 \\
\hline Enclosed Mall & Enclosed mall & 80 & 80 & 78 & 84 & 62 & 77 & 69 & 97 & 84 & 86 & 111 & 95 & 128 & 114 & 144 & 206 \\
\hline Food Sales & Convenience store & 192 & 208 & 193 & 217 & 181 & 198 & 202 & 237 & 214 & 224 & 255 & 231 & 276 & 256 & 298 & 376 \\
\hline Food Sales & Convenience store with gas station & 155 & 168 & 156 & 175 & 146 & 160 & 163 & 191 & 172 & 181 & 205 & 186 & 223 & 206 & 240 & 303 \\
\hline Food Sales & Grocery store/food market & 160 & 174 & 161 & 181 & 151 & 165 & 168 & 197 & 178 & 187 & 212 & 192 & 230 & 213 & 248 & 313 \\
\hline Food Sales & Other food sales & 48 & 53 & 49 & 55 & 46 & 50 & 51 & 60 & 54 & 57 & 64 & 58 & 70 & 64 & 75 & 95 \\
\hline Food Service & Fast food & 374 & 383 & 375 & 396 & 339 & 379 & 361 & 436 & 400 & 405 & 474 & 430 & 519 & 476 & 561 & 710 \\
\hline Food Service & Other food service & 110 & 113 & 110 & 117 & 98 & 111 & 107 & 129 & 118 & 122 & 140 & 127 & 153 & 141 & 166 & 209 \\
\hline Food Service & Restaurant/cafeteria & 202 & 208 & 201 & 215 & 180 & 204 & 196 & 237 & 216 & 222 & 256 & 232 & 279 & 258 & 304 & 383 \\
\hline Inpatient Health Care & Hospital/inpatient health & 202 & 204 & 200 & 201 & 191 & 197 & 186 & 205 & 184 & 193 & 198 & 180 & 203 & 186 & 205 & 237 \\
\hline Laboratory & Laboratory & 249 & 251 & 245 & 256 & 213 & 242 & 221 & 278 & 249 & 252 & 294 & 263 & 323 & 292 & 346 & 460 \\
\hline Lodging & Dormitory/fraternity/sorority & 57 & 61 & 60 & 68 & 45 & 62 & 57 & 83 & 69 & 77 & 94 & 79 & 107 & 95 & 122 & 170 \\
\hline Lodging & Hotel & 71 & 72 & 69 & 74 & 67 & 70 & 69 & 78 & 74 & 75 & 82 & 78 & 87 & 84 & 92 & 107 \\
\hline Lodging & Motel or inn & 79 & 75 & 75 & 73 & 68 & 72 & 66 & 74 & 71 & 68 & 75 & 72 & 80 & 75 & 82 & 98 \\
\hline Lodging & Other lodging & 76 & 72 & 71 & 70 & 65 & 69 & 63 & 70 & 68 & 65 & 72 & 69 & 76 & 71 & 78 & 94 \\
\hline Nursing & Nursing home/assisted living & 117 & 119 & 116 & 121 & 100 & 114 & 104 & 131 & 117 & 119 & 139 & 124 & 152 & 138 & 163 & 217 \\
\hline Office & Administrative/professional office & 56 & 57 & 56 & 59 & 47 & 55 & 48 & 65 & 56 & 58 & 69 & 60 & 77 & 67 & 83 & 116 \\
\hline Office & Bank/other financial & 79 & 81 & 80 & 84 & 66 & 79 & 68 & 92 & 80 & 82 & 98 & 85 & 109 & 96 & 117 & 164 \\
\hline Office & Government office & 69 & 72 & 70 & 74 & 58 & 69 & 59 & 81 & 70 & 72 & 86 & 74 & 95 & 84 & 103 & 144 \\
\hline Office & Medical office (non-di & 47 & 49 & 48 & 51 & 40 & 47 & 41 & 55 & 48 & 49 & 59 & 51 & 65 & 57 & 70 & 99 \\
\hline Office & Mixed-us & 64 & 66 & 65 & 69 & 54 & 64 & 55 & 75 & 65 & 67 & 80 & 69 & 88 & 78 & 96 & 134 \\
\hline Office & Other office & 54 & 55 & 54 & 57 & 45 & 53 & 46 & 63 & 55 & 56 & 66 & 58 & 74 & 65 & 80 & 112 \\
\hline Outpatient Health Care & Clinic/other outpatient health & 72 & 69 & 70 & 69 & 64 & 69 & 57 & 68 & 65 & 60 & 65 & 64 & 67 & 65 & 66 & 75 \\
\hline Outpatient Health Care & Medical office (diagnostic) & 48 & 46 & 46 & 46 & 43 & 46 & 38 & 45 & 43 & 40 & 43 & 42 & 44 & 43 & 44 & 50 \\
\hline Public Assembly & Entertainment/culture & 32 & 33 & 32 & 33 & 28 & 32 & 29 & 36 & 32 & 33 & 38 & 34 & 42 & 38 & 45 & 60 \\
\hline Public Assembly & Library & 85 & 86 & 84 & 88 & 73 & 83 & 76 & 95 & 86 & 87 & 101 & 90 & 111 & 101 & 119 & 158 \\
\hline Public Assembly & Other publ & 39 & 40 & 39 & 41 & 34 & 38 & 35 & 44 & 39 & 40 & 47 & 42 & 51 & 46 & 55 & 73 \\
\hline Public Assembly & Recreation & 37 & 37 & 36 & 38 & 32 & 36 & 33 & 41 & 37 & 37 & 44 & 39 & 48 & 43 & 51 & 68 \\
\hline Public Assembly & Social/meeting & 38 & 39 & 38 & 40 & 33 & 37 & 34 & 43 & 38 & 39 & 45 & 41 & 50 & 45 & 54 & 71 \\
\hline Public Order \& Safety & Fire station/police station & 92 & 92 & 90 & 94 & 78 & 89 & 81 & 102 & 92 & 93 & 108 & 97 & 119 & 108 & 127 & 170 \\
\hline Public Order \& Safety & Other public order and safety & 83 & 84 & 82 & 86 & 71 & 81 & 74 & 93 & 84 & 85 & 99 & 88 & 108 & 98 & 116 & 154 \\
\hline Religious Worship & Religious worship & 33 & 33 & 32 & 34 & 28 & 32 & 29 & 36 & 33 & 33 & 39 & 35 & 42 & 38 & 45 & 60 \\
\hline Retail (except malls) & Other retail & 70 & 71 & 70 & 75 & 53 & 68 & 60 & 85 & 74 & 74 & 96 & 83 & 111 & 98 & 123 & 177 \\
\hline Retail (except malls) & Retail store & 40 & 41 & 40 & 43 & 31 & 39 & 35 & 49 & 42 & 42 & 55 & 48 & 64 & 56 & 71 & 102 \\
\hline Retail (except malls) & Vehicle dealership/showroom & 70 & 72 & 70 & 75 & 54 & 68 & 60 & 85 & 74 & 74 & 97 & 83 & 111 & 99 & 124 & 178 \\
\hline Service & Other service & 84 & 85 & 83 & 87 & 72 & 82 & 75 & 94 & 84 & 85 & 100 & 89 & 109 & 99 & 117 & 156 \\
\hline Service & Post office/postal center & 60 & 60 & 59 & 61 & 51 & 58 & 53 & 67 & 60 & 60 & 70 & 63 & 77 & 70 & 83 & 110 \\
\hline Service & Repair shop & 40 & 40 & 39 & 41 & 34 & 39 & 35 & 44 & 40 & 40 & 47 & 42 & 52 & 47 & 55 & 73 \\
\hline Service & Vehicle service/repair shop & 46 & 46 & 45 & 47 & 39 & 45 & 41 & 51 & 46 & 47 & 54 & 49 & 60 & 54 & 64 & 85 \\
\hline Service & Vehicle storage/maintenance & 20 & 20 & 20 & 21 & 17 & 19 & 18 & 22 & 20 & 20 & 24 & 21 & 26 & 23 & 28 & 37 \\
\hline Strip Shopping Mall & Strip shopping mall & 84 & 84 & 82 & 89 & 65 & 81 & 73 & 102 & 88 & 91 & 117 & 100 & 135 & 120 & 151 & 216 \\
\hline Warehouse & Distribution/shipping center & 18 & 22 & 24 & 28 & 15 & 25 & 21 & 38 & 32 & 31 & 51 & 43 & 70 & 58 & 86 & 161 \\
\hline Warehouse & Non-refrigerated warehouse & 8 & 11 & 11 & 14 & 7 & 12 & 10 & 19 & 16 & 15 & 25 & 21 & 34 & 28 & 41 & 78 \\
\hline Warehouse & Refrigerated warehouse & 96 & 97 & 95 & 99 & 82 & 94 & 86 & 108 & 96 & 98 & 114 & 102 & 125 & 113 & 134 & 178 \\
\hline Residential & Mobile/manufactured home & 54 & 57 & 57 & 64 & 42 & 58 & 54 & 78 & 65 & 73 & 88 & 74 & 101 & 89 & 115 & 160 \\
\hline Residential & Single-family detached & 40 & 42 & 42 & 47 & 31 & 43 & 40 & 58 & 48 & 54 & 65 & 55 & 75 & 66 & 85 & 119 \\
\hline Residential & Single-family attached & 46 & 49 & 49 & 54 & 36 & 50 & 46 & 66 & 55 & 62 & 75 & 63 & 86 & 76 & 98 & 137 \\
\hline Residential & Apartment (in 2-4 unit building) & 68 & 72 & 71 & 80 & 53 & 73 & 68 & 97 & 81 & 91 & 110 & 93 & 126 & 112 & 144 & 201 \\
\hline Residential & Apartment (in $5+$ unit building) & 46 & 49 & 48 & 54 & 36 & 50 & 46 & 66 & 55 & 62 & 75 & \begin{tabular}{|l|}
63 \\
\end{tabular} & \begin{tabular}{|l|}
86 \\
\end{tabular} & 76 & 98 & 136 \\
\hline & & & & & & & & & & & & & & & & & \\
\hline
\end{tabular}


Table 2. Energy Use Intensity Targets

\begin{tabular}{|c|c|c|c|c|c|c|c|c|c|c|c|c|c|c|c|c|c|}
\hline \multirow[b]{3}{*}{ No. } & \multirow[b]{3}{*}{ Commercial Building Type } & \multicolumn{16}{|c|}{ EUls by Building Type by Climate Zone (kBtu/sf-yr) } \\
\hline & & \multicolumn{16}{|c|}{ ASHRAE Climate Zone } \\
\hline & & $1 \mathrm{~A}$ & 2A & 2B & 3A & 3B & 3B & $3 C$ & 4A & 4B & 4C & $5 A$ & 5B & $6 \mathrm{~A}$ & 6B & 7 & 8 \\
\hline 1 & Admin/professional office & 39 & 40 & 39 & 42 & 33 & 39 & 33 & 46 & 40 & 40 & 48 & 42 & 54 & 47 & 58 & 81 \\
\hline 2 & Bank/other financial & 55 & 57 & 56 & 59 & 46 & 55 & 47 & 65 & 56 & 57 & 68 & 59 & 76 & 67 & 82 & 115 \\
\hline 3 & Government office & 49 & 50 & 49 & 52 & 41 & 48 & 42 & 57 & 49 & 50 & 60 & 52 & 67 & 59 & 72 & 101 \\
\hline 4 & Medical office (non-diag) & 33 & 34 & 33 & 35 & 28 & 33 & 28 & 39 & 34 & 34 & 41 & 36 & 46 & 40 & 49 & 69 \\
\hline 5 & Mixed-use office & 45 & 46 & 45 & 48 & 38 & 45 & 39 & 53 & 46 & 47 & 56 & 48 & 62 & 55 & 67 & 94 \\
\hline 6 & Other office & 38 & 39 & 38 & 40 & 32 & 37 & 32 & 44 & 38 & 39 & 47 & 40 & 52 & 46 & 56 & 78 \\
\hline 7 & Laboratory & 178 & 176 & 171 & 175 & 147 & 165 & 159 & 194 & 173 & 179 & 209 & 187 & 232 & 211 & 249 & 331 \\
\hline 8 & Distribution/ship center & 12 & 16 & 16 & 20 & 11 & 18 & 14 & 27 & 23 & 22 & 36 & 30 & 49 & 40 & 60 & 113 \\
\hline 9 & Non-refrig warehouse & 6 & 8 & 8 & 10 & 5 & 9 & 7 & 13 & 11 & 11 & 17 & 14 & 24 & 19 & 29 & 54 \\
\hline 10 & Convenience store & 135 & 146 & 135 & 152 & 127 & 139 & 141 & 166 & 150 & 157 & 178 & 162 & 193 & 179 & 208 & 263 \\
\hline 11 & Convenience store+gas & 108 & 118 & 109 & 122 & 102 & 112 & 114 & 133 & 121 & 126 & 144 & 130 & 156 & 144 & 168 & 212 \\
\hline 12 & Grocery/food market & 112 & 122 & 113 & 127 & 106 & 116 & 118 & 138 & 125 & 131 & 149 & 135 & 161 & 149 & 174 & 219 \\
\hline 13 & Other food sales & 34 & 37 & 34 & 38 & 32 & 35 & 36 & 42 & 38 & 40 & 45 & 41 & 49 & 45 & 53 & 66 \\
\hline 14 & Fire/police station & 66 & 65 & 63 & 64 & 54 & 61 & 59 & 71 & 64 & 66 & 77 & 69 & 85 & 78 & 92 & 122 \\
\hline 15 & Other public order/safety & 60 & 59 & 57 & 59 & 49 & 55 & 53 & 65 & 58 & 60 & 70 & 63 & 78 & 71 & 84 & 111 \\
\hline 16 & Medical office (diagnostic) & 33 & 32 & 32 & 32 & 30 & 32 & 27 & 32 & 30 & 28 & 30 & 30 & 31 & 30 & 31 & 35 \\
\hline 17 & Clinic/other outpatient health & 50 & 48 & 49 & 48 & 45 & 48 & 40 & 48 & 46 & 42 & 46 & 45 & 47 & 45 & 46 & 52 \\
\hline 18 & Refrigerated warehouse & 69 & 68 & 66 & 68 & 57 & 64 & 62 & 75 & 67 & 69 & 81 & 72 & 90 & 82 & 96 & 128 \\
\hline 19 & Religious worship & 23 & 23 & 22 & 23 & 19 & 22 & 21 & 25 & 23 & 23 & 27 & 25 & 30 & 28 & 33 & 43 \\
\hline 20 & Entertainment/culture & 23 & 23 & 22 & 23 & 19 & 21 & 21 & 25 & 23 & 23 & 27 & 24 & 30 & 28 & 32 & 43 \\
\hline 21 & Library & 61 & 61 & 59 & 60 & 50 & 57 & 55 & 67 & 60 & 61 & 72 & 64 & 80 & 73 & 86 & 114 \\
\hline 22 & Recreation & 26 & 26 & 25 & 26 & 22 & 24 & 24 & 29 & 26 & 26 & 31 & 28 & 34 & 31 & 37 & 49 \\
\hline 23 & Social/meeting & 28 & 27 & 26 & 27 & 23 & 26 & 25 & 30 & 27 & 28 & 32 & 29 & 36 & 33 & 39 & 51 \\
\hline 24 & Other public assembly & 28 & 28 & 27 & 28 & 23 & 26 & 25 & 31 & 27 & 28 & 33 & 30 & 37 & 33 & 39 & 52 \\
\hline 25 & College/university & 62 & 61 & 60 & 62 & 45 & 58 & 50 & 72 & 60 & 65 & 78 & 65 & 90 & 78 & 99 & 147 \\
\hline 26 & Elementary/middle school & 38 & 37 & 36 & 37 & 30 & 35 & 32 & 41 & 36 & 36 & 42 & 37 & 46 & 41 & 49 & 72 \\
\hline 27 & High school & 45 & 45 & 44 & 46 & 33 & 42 & 37 & 52 & 44 & 47 & 57 & 48 & 66 & 57 & 72 & 107 \\
\hline 28 & Preschool/daycare & 49 & 48 & 46 & 48 & 39 & 45 & 41 & 52 & 46 & 47 & 54 & 47 & 60 & 53 & 63 & 93 \\
\hline 29 & Other classroom education & 25 & 25 & 25 & 25 & 18 & 24 & 21 & 29 & 25 & 26 & 32 & 27 & 37 & 32 & 40 & 60 \\
\hline 30 & Fast food & 261 & 268 & 263 & 277 & 237 & 266 & 253 & 305 & 280 & 284 & 332 & 301 & 364 & 333 & 393 & 497 \\
\hline 31 & Restaurant/cafeteria & 141 & 145 & 141 & 150 & 126 & 143 & 137 & 166 & 151 & 156 & 179 & 163 & 195 & 181 & 213 & 268 \\
\hline 32 & Other food service & 77 & 79 & 77 & 82 & 69 & 78 & 75 & 91 & 83 & 85 & 98 & 89 & 107 & 99 & 116 & 146 \\
\hline 33 & Hospital/inpatient health & 142 & 143 & 140 & 141 & 134 & 138 & 130 & 143 & 129 & 135 & 139 & 126 & 142 & 130 & 144 & 166 \\
\hline 34 & Nursing home/assisted living & 84 & 83 & 81 & 83 & 69 & 78 & 75 & 91 & 82 & 84 & 99 & 88 & 109 & 100 & 118 & 156 \\
\hline 35 & Dormitory/fraternity/sorority & 40 & 43 & 42 & 47 & 31 & 43 & 40 & 58 & 48 & 54 & 65 & 55 & 75 & 66 & 85 & 119 \\
\hline 36 & Hotel & 50 & 51 & 48 & 52 & 47 & 49 & 48 & 55 & 52 & 52 & 57 & 55 & 61 & 59 & 65 & 75 \\
\hline 37 & Motel or inn & 55 & 53 & 52 & 51 & 48 & 50 & 46 & 52 & 50 & 48 & 53 & 50 & 56 & 52 & 57 & 69 \\
\hline 38 & Other lodging & 53 & 50 & 50 & 49 & 46 & 48 & 44 & 49 & 48 & 46 & 50 & 48 & 53 & 50 & 55 & 66 \\
\hline 39 & Vehicle dealership/showroom & 49 & 50 & 49 & 53 & 38 & 48 & 42 & 60 & 52 & 52 & 68 & 58 & 78 & 69 & 87 & 124 \\
\hline 40 & Retail store & 28 & 29 & 28 & 30 & 21 & 27 & 24 & 34 & 30 & 30 & 39 & 33 & 45 & 40 & 50 & 71 \\
\hline 41 & Other retail & 49 & 50 & 49 & 52 & 37 & 48 & 42 & 59 & 52 & 52 & 67 & 58 & 78 & 69 & 86 & 124 \\
\hline 42 & Post office/postal center & 43 & 42 & 41 & 42 & 35 & 39 & 38 & 46 & 41 & 43 & 50 & 45 & 56 & 51 & 60 & 79 \\
\hline 43 & Repair shop & 28 & 28 & 27 & 28 & 23 & 26 & 25 & 31 & 28 & 28 & 33 & 30 & 37 & 34 & 40 & 53 \\
\hline 44 & Vehicle service/repair shop & 33 & 33 & 32 & 32 & 27 & 31 & 29 & 36 & 32 & 33 & 39 & 35 & 43 & 39 & 46 & 61 \\
\hline 45 & Vehicle storage/maintenance & 14 & 14 & 14 & 14 & 12 & 13 & 13 & 16 & 14 & 14 & 17 & 15 & 19 & 17 & 20 & 27 \\
\hline 46 & Other service & 60 & 60 & 58 & 59 & 50 & 56 & 54 & 65 & 59 & 60 & 71 & 63 & 78 & 71 & 84 & 112 \\
\hline 47 & Strip shopping mall & 59 & 59 & 58 & 62 & 46 & 57 & 51 & 71 & 62 & 63 & 82 & 70 & 94 & 84 & 106 & 151 \\
\hline \multirow[t]{3}{*}{48} & Enclosed mall & 56 & 56 & 55 & 59 & 44 & 54 & 49 & 68 & 59 & 60 & 78 & 67 & 90 & 80 & 101 & 144 \\
\hline & & \multicolumn{16}{|c|}{ ASHRAE Climate Zone } \\
\hline & Residential Building Type & 1A & 2A & 2B & 3A & 3B & 3B & $3 C$ & 4A & 4B & $4 \mathrm{C}$ & $5 \mathbf{A}$ & 5B & $6 \mathrm{~A}$ & 6B & 7 & 8 \\
\hline 49 & Mobile home & 38 & 40 & 40 & 45 & 30 & 41 & 38 & 54 & 45 & 51 & 62 & 52 & 71 & 62 & 80 & 112 \\
\hline 50 & SF-detached & 28 & 30 & 30 & 33 & 22 & 30 & 28 & 40 & 34 & 38 & 46 & 38 & 52 & 46 & 60 & 83 \\
\hline 51 & SF-attached & 32 & 34 & 34 & 38 & 25 & 35 & 32 & 46 & 39 & 43 & 53 & 44 & 60 & 53 & 69 & 96 \\
\hline 52 & Apartment building (2-4 units) & 47 & 50 & 50 & 56 & 37 & 51 & 47 & 68 & 57 & 64 & 77 & 65 & 89 & 78 & 101 & 140 \\
\hline 53 & Apartment building (5+ units) & 32 & 34 & 34 & 38 & 25 & 35 & 32 & 46 & 39 & 43 & 53 & 44 & 60 & 53 & 68 & 96 \\
\hline
\end{tabular}


Table 3: Operating Shift Multipliers

\begin{tabular}{|c|c|c|c|c|}
\hline \multicolumn{5}{|c|}{ Operating Shift Multipliers } \\
\hline \multirow[b]{2}{*}{ No. } & \multirow[b]{2}{*}{ Building Activity/Type } & \multicolumn{3}{|c|}{ Weekly Hours } \\
\hline & & $\begin{array}{l}50 \text { or } \\
\text { less }\end{array}$ & $\begin{array}{c}51 \text { to } \\
167\end{array}$ & 168 \\
\hline 1 & Admin/professional office & 1.0 & 1.0 & 1.4 \\
\hline 2 & Bank/other financial & 1.0 & 1.0 & 1.4 \\
\hline 3 & Government office & 1.0 & 1.0 & 1.4 \\
\hline 4 & Medical office (non-diag) & 1.0 & 1.0 & 1.4 \\
\hline 5 & Mixed-use office & 1.0 & 1.0 & 1.4 \\
\hline 6 & Other office & 1.0 & 1.0 & 1.4 \\
\hline 7 & Laboratory & 1.0 & 1.0 & 1.0 \\
\hline 8 & Distribution/ship center & 0.7 & 1.4 & 2.1 \\
\hline 9 & Non-refrig warehouse & 0.7 & 1.4 & 2.1 \\
\hline 10 & Convenience store & 1.0 & 1.0 & 1.4 \\
\hline 11 & Convenience store+gas & 1.0 & 1.0 & 1.4 \\
\hline 12 & Grocery/food market & 1.0 & 1.0 & 1.4 \\
\hline 13 & Other food sales & 1.0 & 1.0 & 1.4 \\
\hline 14 & Fire/police station & 0.8 & 0.8 & 1.1 \\
\hline 15 & Other public order/safety & 0.8 & 0.8 & 1.1 \\
\hline 16 & Medical office (diagnostic) & 1.0 & 1.0 & 1.5 \\
\hline 17 & Clinic/other outpatient health & 1.0 & 1.0 & 1.5 \\
\hline 18 & Refrigerated warehouse & 1.0 & 1.0 & 1.0 \\
\hline 19 & Religious worship & 0.9 & 1.7 & 1.7 \\
\hline 20 & Entertainment/culture & 0.8 & 1.5 & 1.5 \\
\hline 21 & Library & 0.8 & 1.5 & 1.5 \\
\hline 22 & Recreation & 0.8 & 1.5 & 1.5 \\
\hline 23 & Social/meeting & 0.8 & 1.5 & 1.5 \\
\hline 24 & Other public assembly & 0.8 & 1.5 & 1.5 \\
\hline 25 & College/university & 0.8 & 1.3 & 1.3 \\
\hline 26 & Elementary/middle school & 0.8 & 1.3 & 1.3 \\
\hline 27 & High school & 0.8 & 1.3 & 1.3 \\
\hline
\end{tabular}

\begin{tabular}{|c|c|c|c|c|}
\hline \multicolumn{5}{|c|}{ Operating Shift Multipliers } \\
\hline \multirow[b]{2}{*}{ No. } & \multirow[b]{2}{*}{ Building Activity/Type } & \multicolumn{3}{|c|}{ Weekly Hours } \\
\hline & & $\begin{array}{l}50 \text { or } \\
\text { less }\end{array}$ & \begin{tabular}{|c|}
51 to \\
167
\end{tabular} & 168 \\
\hline 28 & Preschool/daycare & 0.8 & 1.3 & 1.3 \\
\hline 29 & Other classroom education & 0.8 & 1.3 & 1.3 \\
\hline 30 & Fast food & 0.4 & 1.1 & 2.1 \\
\hline 31 & Restaurant/cafeteria & 0.4 & 1.1 & 2.1 \\
\hline 32 & Other food service & 0.4 & 1.1 & 2.1 \\
\hline 33 & Hospital/inpatient health & 1.0 & 1.0 & 1.0 \\
\hline 34 & Nursing home/assisted living & 1.0 & 1.0 & 1.0 \\
\hline 35 & Dormitory/fraternity/sorority & 1.0 & 1.0 & 1.0 \\
\hline 36 & Hotel & 1.0 & 1.0 & 1.0 \\
\hline 37 & Motel or inn & 1.0 & 1.0 & 1.0 \\
\hline 38 & Other lodging & 1.0 & 1.0 & 1.0 \\
\hline 39 & Vehicle dealership/showroom & 0.8 & 1.2 & 1.8 \\
\hline 40 & Retail store & 0.8 & 1.2 & 1.8 \\
\hline 41 & Other retail & 0.8 & 1.2 & 1.8 \\
\hline 42 & Post office/postal center & 0.7 & 1.5 & 1.5 \\
\hline 43 & Repair shop & 0.7 & 1.5 & 1.5 \\
\hline 44 & Vehicle service/ & 0.7 & 1.5 & 1.5 \\
\hline 45 & Vehicle storas & 0.7 & 1.5 & 1.5 \\
\hline 46 & Othe & 0.7 & 1.5 & 1.5 \\
\hline 47 & Strip s & 1.0 & 1. & 1.0 \\
\hline 48 & Enclosed mall & 1.0 & 1.0 & 1.0 \\
\hline \multicolumn{5}{|c|}{ Residential Building Activity/Type } \\
\hline 49 & Mobile home & 1.0 & 1.0 & 1.0 \\
\hline 50 & SF-detached & 1.0 & 1.0 & 1.0 \\
\hline 51 & SF-attached & 1.0 & 1.0 & 1.0 \\
\hline 52 & Apartment builc & 1.0 & 1.0 & 1.0 \\
\hline 53 & Apartment building (5+ units) & 1.0 & 1.0 & 1.0 \\
\hline
\end{tabular}

